\title{
Intramedullary versus extramedullary fixation in the management of subtrochanteric femur fractures: a meta-analysis
}

\author{
Pengcheng Liu ${ }^{1,2, *}$ \\ Xing $\mathrm{Wu}^{1, *}$ \\ Hui Shi ${ }^{1,2}$ \\ Run Liu' \\ Hexi Shu' \\ JinPeng Gong' \\ Yong Yang' \\ Qi Sun' \\ Jiezhou $\mathrm{Wu}^{1,2}$ \\ Xiaoyang $\mathrm{Nie}^{1}$ \\ Ming Cai' \\ 'Department of Orthopedics, \\ Shanghai Tenth People's Hospital, \\ Tongji University, School of Medicine, \\ Shanghai, ${ }^{2}$ First Clinical Medical \\ College, Nanjing Medical University, \\ Nanjing, People's Republic of China \\ *These authors contributed equally \\ to this work
}

This article was published in the following Dove Press journal:

Clinical Interventions in Aging

28 April 2015

Number of times this article has been viewed
Background: Intramedullary and extramedullary fixation methods are used in the management of subtrochanteric femur fractures. However, whether intramedullary or extramedullary fixation is the primary treatment for subtrochanteric femur fractures in adults remains debatable.

Level of evidence: Meta-analyses of prospective studies, level I.

Materials and methods: The Cochrane library, Embase, Google Scholar, and PubMed databases were searched separately for all relevant studies published before January 1, 2015. No language restriction was applied. Prospective randomized controlled trials that compared intramedullary or extramedullary internal fixation to repair subtrochanteric femur fractures in adults were included. We determined intraoperative data, postoperative complications, fracture fixation complications, wound infection, hospital stay days, and final outcome measures to assess the relative effects of different internal fixation methods for the treatment of subtrochanteric femur fractures in adults.

Results: Six studies were included in our meta-analysis. The relative risks (RRs) of revision rate was $83 \%$ lower (RR, $0.17,95 \%$ confidence interval [CI], 0.05 to $0.60 ; P=0.006$ ), fixation failure rate was $64 \%$ lower (RR, $0.36,95 \% \mathrm{CI}, 0.12$ to $1.08 ; P=0.07$ ), non-union rate was $77 \%$ lower (RR, $0.23,95 \% \mathrm{CI}, 0.07$ to $0.81 ; P=0.02$ ) in the intramedullary group compared with the extramedullary group. No significant differences were found between the intramedullary group and extramedullary group for intraoperative data, postoperative complications, wound infection, hospital stay days or final outcome measures.

Conclusion: In conclusion, our meta-analysis suggests that there was no significant difference in intraoperative data, postoperative complications, wound infection, hospital stay days or final outcome measures between intramedullary and extramedullary internal fixation. However, a significant decrease occurred in the rate of fracture fixation complications for patients treated with intramedullary internal fixation, especially in elderly patients. Some differences were not significant, but the treatment of elderly subtrochanteric femur fractures using intramedullary internal fixation is recommended.

Keywords: subtrochanteric femur fracture, extramedullary, intramedullary, systematic review, meta-analysis

\section{Introduction}

Subtrochanteric femur fractures are common in elderly patients. The management of subtrochanteric femur fractures remains challenging for surgeons because of the focus of the muscles that act on the distal and proximal fragments and the inherent instability of the pattern. Operative alternatives for subtrochanteric femur fractures differ worldwide but primarily include intramedullary and extramedullary internal fixation. Prevention of the complications of long-time immobilization and the beginning
Department of Orthopedics, Shanghai

Tenth People's Hospital, No 30I Middle

Yanchang Road, Shanghai 200072,

People's Republic of China

Tel +86I326222 76I3

Email cmdoctor@tongji.edu.cn 
of early mobilization becomes increasingly important for subtrochanteric femur fractures.

Intramedullary and extramedullary internal fixation devices are now advocated for the management of subtrochanteric femur fractures. ${ }^{1}$ Surgeons and patients must consider many factors to determine the preferred management, and it is debatable whether intramedullary or extramedullary internal fixation is more appropriate for the treatment of subtrochanteric femur fractures in adult patients. ${ }^{2}$ Biomechanical studies suggest that intramedullary internal fixation provides a stable construct. ${ }^{3-7}$ Some studies focused on different types of intramedullary internal fixations. ${ }^{3,5}$ Three studies compared the stability between plates and nails. ${ }^{3,6,7}$

Therefore, we conducted a new meta-analysis of all prospective randomized controlled trials (RCTs) to evaluate intraoperative data (which included operative time, fluoroscopy time, intraoperative blood loss, number of patients receiving blood, and blood transfused units), postoperative complications (which included hematoma and general complications, such as heart failure), fracture fixation complications (which included revision rate, fixation failure rate, non-union rate, and femoral neck fracture rate), femoral neck fracture was defined as femoral shaft or neck fracture during surgery (fracture was detected using fluoroscopy control or a new fracture at postoperative radiographic control), ${ }^{8}$ wound infection (which included deep wound infection and superficial wound infection), hospital stay days, and final outcome measures (which included mortality, hip mobility, hip/thigh pain score, Parker and Palmer score, union time, return home rate, and regain independent mobility) in adult patients treated with either intramedullary or extramedullary internal fixation for subtrochanteric femur fractures.

\section{Materials and methods Search strategy and criteria}

Two investigators (PCL and HS) independently and separately searched the PubMed, Cochrane library, Google Scholar, and Embase databases to retrieve all relevant studies published before January 1, 2015. The search strategy was based on combinations of medical subject headings (MeSH) and the keywords "Femur Fracture", "Subtrochanteric Fractures", and "Fractures, Subtrochanteric". No restrictions to specific languages or years of publication were imposed. The "related articles" function was also used to broaden our search. Two investigators (PCL and XW) manually examined the entire reference lists of these selected studies to identify all possible relevant studies that were not discovered during the previous database searches. The corresponding authors were contacted only when additional information of the studies was needed.

\section{Inclusion criteria}

1. Prospective studies with high level I evidence.

2. Internal fixation of intertrochanteric hip fractures with subtrochanteric extension or subtrochanteric fractures.

3. Included both intramedullary and extramedullary implants.

4. Greater than a 6-month minimum follow-up.

5. Follow-up examinations that included at least one of the following outcome measurements: operative time, blood loss, number of patients transfused, intraoperative complications, postoperative complications, wound complications, rate of internal fixation failure, rate of non-union, entire hospital stay days, and final functional recovery.

\section{Exclusion criteria}

1. Retrospective studies.

2. Levels II, III, or IV evidence.

3. Less than a 6-month minimum follow-up.

4. Only report either intramedullary or extramedullary implants.

5. Did not report the standard differentiation of all interesting data.

6. Pure intracapsular and intertrochanteric fractures.

7. Any pathological fractures.

\section{Data extraction}

Two readers (PCL and HS) reviewed titles and abstracts using the above mentioned selection criteria. Two readers also independently performed data extraction of all possible variables and outcomes of interest and the assessment of methodological quality. Any disagreement was resolved by discussion and consensus. The methodological quality of all trials was also assessed using the Cochrane Handbook for Systematic Reviews of Interventions 5.1.0.

\section{Outcomes}

All eligible studies were carefully reviewed for baseline data, intervention methods, and outcome measures. Both subjective and objective functional outcome measurements were fully used to evaluate the data. The following measures were carefully reviewed in all studies: operative time, blood loss, number of patients transfused, intraoperative complications, postoperative complications, wound complications, rate of 
internal fixation failure, rate of non-union, entire hospital stay days, and final functional recovery. If the studies reported several different functional outcome results at the different follow-up visits, the results after different follow-up visits were used for the study. Appropriate recommendations for the management of subtrochanteric fractures were made according to the pooled measurements of the highest level evidence.

\section{Statistical analysis}

The statistical analysis was performed completely in Review Manager (RevMan), version 5.3. Copenhagen: The Nordic Cochrane Centre, The Cochrane Collaboration, 2014. The relative risks (RRs) for the dichotomous variables were measured using 95\% confidence intervals (CIs). Also the weighted mean difference (WMD) was measured using $95 \%$ CIs for continuous variables. $P$-values $<0.05$ were considered statistically significant, and the $95 \%$ CIs were also reported. Statistical heterogeneity between studies was evaluated using the $Q$-statistic and quantified using the $I^{2}$ statistic. Fixed-effects models and random-effects models were commonly used to obtain summary RRs or WMDs. If the $Q$ or $I^{2}$ statistic value was significant, then the random-effects model was used. Otherwise, the fixedeffects model was used. Funnel plots and Egger's test (with $P<0.05$ considered statistically significant) were created to visually evaluate for the presence of publication bias. A sensitivity analysis was also conducted, in which the RCTs were excluded to determine the stability of the combined RRs or WMDs.

\section{Results}

\section{Literature search}

An initial 13,767 articles were identified. After 13,268 duplicates and unrelated articles were excluded, 499 articles were assessed for detailed evaluation. A careful screening of all titles excluded 483 articles that did not pertain to the topic of interest. An abstract review excluded eight more articles (five retrospective studies, two laboratory studies, and one review), which left eight studies for further full publication review. Only one study was excluded because the article did not report the standard differentiation of the data. ${ }^{9}$ One additional study was excluded because the article was a prospective non-RCT. ${ }^{10}$ Therefore, six studies met the selection criteria and were completely suitable for our meta-analysis. ${ }^{8,11-15}$ All studies were prospective RCTs (Figure 1). A total of 232 patients (123 intramedullary and 109 extramedullary) were enrolled in our studies. The key

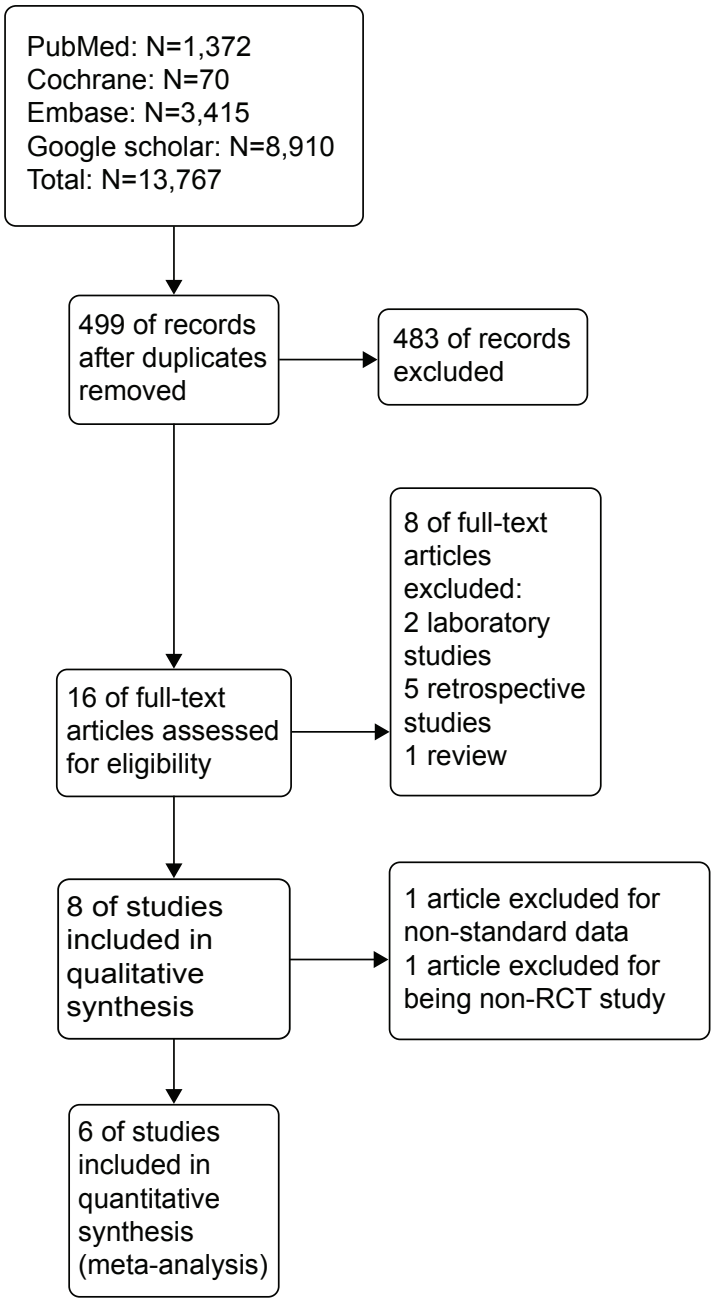

Figure I The selection of randomized controlled trials (RCTs) comparing intramedullary with extramedullary fixation for subtrochanteric fractures in adults is shown.

characteristics of all included studies are summarized in Table 1. All of the studies involved patients with subtrochanteric fractures and were followed-up for at least 12 months. Six level I RCT studies from 1994 to 2007 that compared intramedullary nails with extramedullary plates for the treatment of subtrochanteric fractures prospectively and randomly were identified. ${ }^{8,11-15}$ Three of the studies included unstable intertrochanteric fractures, and these data were specially provided in a separate subgroup..$^{8,11,14}$ Only one level I study examined intertrochanteric fractures with the subtrochanteric extension. ${ }^{12}$ Only one RCT included young adult patients. ${ }^{13}$ There was complete agreement $(100 \%)$ between the two independent reviewers for the entire data extraction. Figure 2 summarizes the methodological quality of all studies. All of the studies were RCTs, with a high level of methodological quality. Therefore, the methodological bias of this study was very low. 
Table I Summary of demographic data for eligible studies

\begin{tabular}{|c|c|c|c|c|c|c|c|c|c|}
\hline Study & Year & $\begin{array}{l}\text { Study } \\
\text { type }\end{array}$ & $\begin{array}{l}\text { Level of } \\
\text { study }\end{array}$ & Implants & $\begin{array}{l}\text { Follow-up } \\
\text { (months) }\end{array}$ & $\begin{array}{l}\text { Rate of } \\
\text { follow-up }\end{array}$ & $\begin{array}{l}\text { Sample } \\
\text { size }\end{array}$ & $\begin{array}{l}\text { Mean age } \\
\text { (years) }\end{array}$ & $\begin{array}{l}\text { Female } \\
\text { (\%) }\end{array}$ \\
\hline \multirow[t]{2}{*}{ Ekstrom et al ${ }^{8}$} & 2007 & RCT & I & PFN & 12 & 100 & 18 & 77 & 85 \\
\hline & & & & MSP & & & 13 & 83 & 79 \\
\hline \multirow[t]{2}{*}{ Miedel et al" } & 2005 & $\mathrm{RCT}$ & I & GN & 12 & $67.2^{*}$ & 16 & $84^{*}$ & $81^{*}$ \\
\hline & & & & MSP & & & 12 & & \\
\hline \multirow[t]{2}{*}{ Sadowski et al ${ }^{12}$} & 2002 & RCT & I & GN & 12 & 90 & 20 & 80 & 69.2 \\
\hline & & & & DCS & & & 19 & 77 & 73.7 \\
\hline \multirow[t]{2}{*}{ Lee et $\mathrm{al}^{13}$} & 2007 & RCT & I & RTRN & 28.9 & 100 & 34 & 35.4 & 26.5 \\
\hline & & & & DCS & 27.2 & & 32 & 36.8 & 18.8 \\
\hline \multirow[t]{2}{*}{ Goldhagen et al ${ }^{14}$} & 1994 & RCT & I & GN & 6.4 & 100 & 6 & $78 *$ & $69.4^{*}$ \\
\hline & & & & $\mathrm{CHS}$ & & & 4 & & \\
\hline \multirow[t]{2}{*}{ Rahme and Harris ${ }^{15}$} & 2007 & RCT & I & PFN & 12 & 70.7 & 29 & 73 & 55.2 \\
\hline & & & & $\mathrm{BP}$ & & & 29 & 67 & 58.6 \\
\hline
\end{tabular}

Note: *Data include demographics for entire study (including pertrochanteric fractures).

Abbreviations: PFN, proximal femoral nail; MSP, medoff sliding plate; GN, gamma nail; DCS, dynamic condylar screw; RTRN, Russell-Taylor reconstruction nail; CHS, compression hip screw; BP, 95-degree blade plate; RCT, randomized controlled trial.

\section{Main analysis}

Table 2 summarizes all outcomes of this meta-analysis. No significant differences were found between the intramedullary group and the extramedullary group for the intraoperative data when all of the patients were pooled into the meta-analysis: operative time (minutes) (WMD, -31.60,

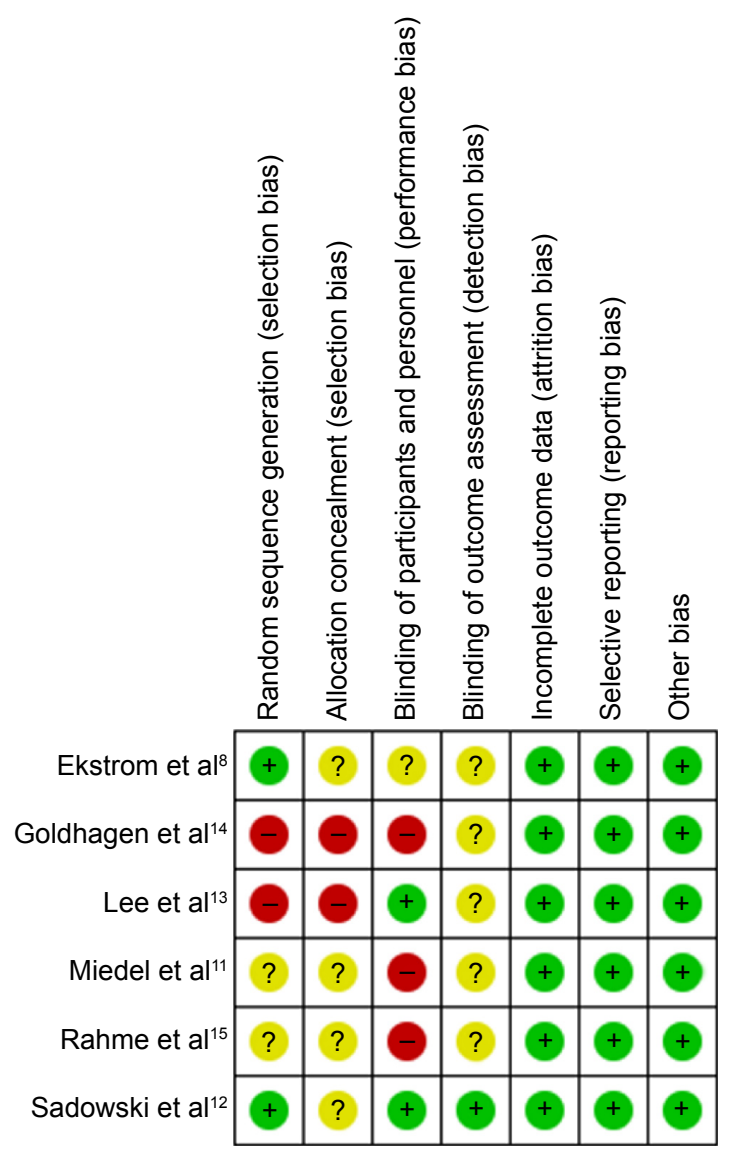

Figure 2 Risk of bias summary: review of authors' judgments of each risk of bias item for each included study.

Notes: + represents yes; - represents no; ? represents not clear.
95\% CI, -70.86 to $7.66 ; P=0.11$ ), fluoroscopy time (minutes) (WMD, $1.32,95 \% \mathrm{CI},-0.45$ to $3.09 ; P=0.14$ ), intraoperative blood loss (mL) (WMD, $-43.21,95 \% \mathrm{CI},-486.11$ to 399.70; $P=0.85$ ), number of patients receiving blood (RR, $1.14,95 \% \mathrm{CI}, 0.26$ to $5.12 ; P=0.86$ ), and blood transfused (units) (WMD, $-0.42,95 \% \mathrm{CI},-2.37$ to $1.54 ; P=0.68$ ). The random-effects model was used because no significant clinical heterogeneity for intraoperative data was observed between the studies.

There were no significant differences in postoperative complications between the intramedullary group and the extramedullary group, including general complications (RR, $1.22,95 \% \mathrm{CI}, 0.57$ to $2.62 ; P=0.61$ ) and hematoma (RR, not estimable). The random-effects model was used because no significant clinical heterogeneity in postoperative complications was observed between the studies.

The RR of revision rate was $83 \%$ lower in the intramedullary group compared to the extramedullary group (RR, $0.17,95 \%$ CI, 0.05 to $0.60 ; P=0.006$ ) (Figure 3 ) for elderly patients. The RR of fixation failure rate was $64 \%$ lower in the intramedullary group compared with the extramedullary group (RR, $0.36,95 \% \mathrm{CI}, 0.12$ to $1.08 ; P=0.07$ ) (Figure 4) for all patients, and the RR was $76 \%$ lower in the intramedullary group (RR, $0.24,95 \% \mathrm{CI}, 0.07$ to $0.89 ; P=0.03$ ) (Figure 4) for elderly patients. The RR of non-union rate was $77 \%$ lower in the intramedullary group compared to the extramedullary group (RR, $0.23,95 \% \mathrm{CI}, 0.07$ to $0.81 ; P=0.02$ ) (Figure 5), and it was $78 \%$ lower in the intramedullary group (RR, 0.22 , $95 \%$ CI, 0.06 to $0.86 ; P=0.03$ ) (Figure 5) for elderly patients. No significant difference was observed for femoral neck fracture rate (RR, $2.50,95 \% \mathrm{CI}, 0.27$ to $23.04 ; P=0.42$ ). No significant heterogeneity was observed among these studies (revision, $P=0.26, I^{2}=21 \%$; fixation failure, $P=0.20, I^{2}=35 \%$; 


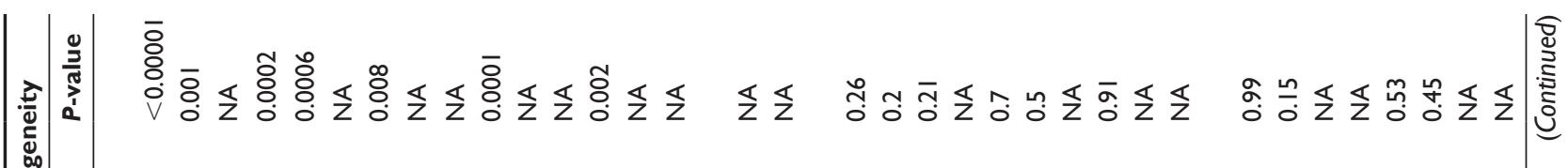

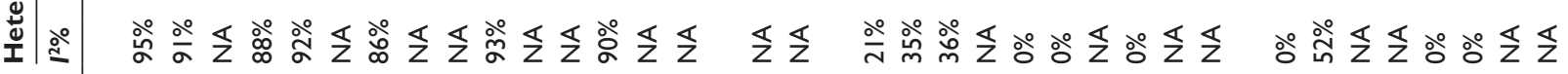

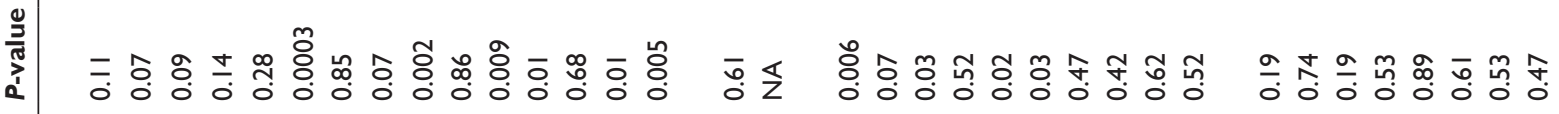

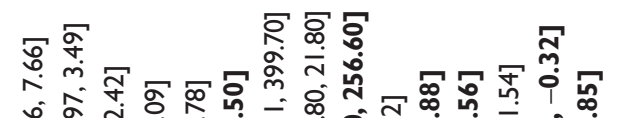

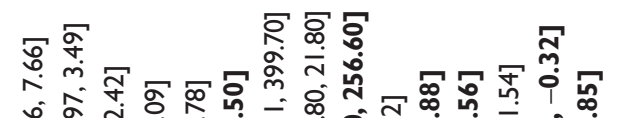

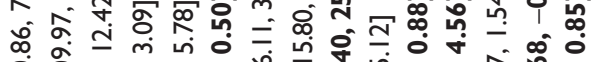

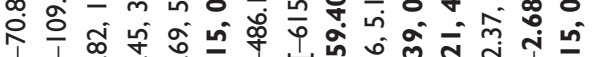

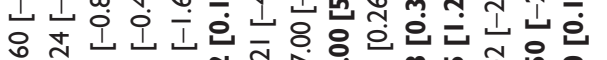

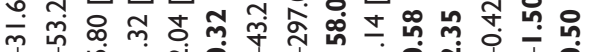

:

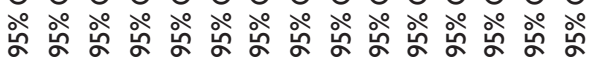

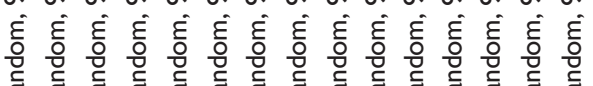

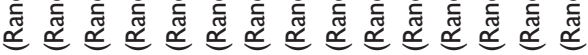

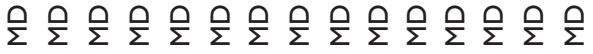

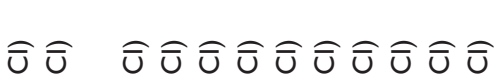

ิิธิิิ

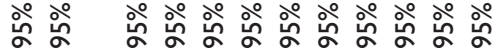

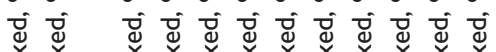

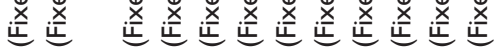

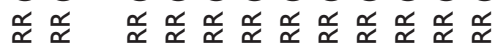

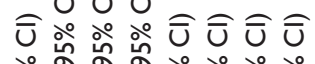

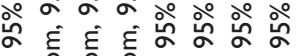

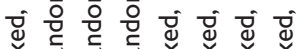

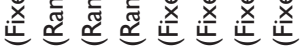

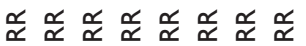

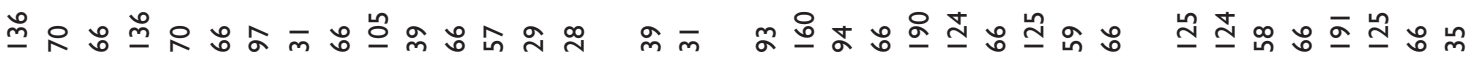

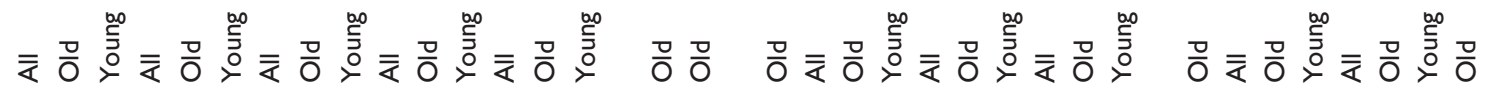

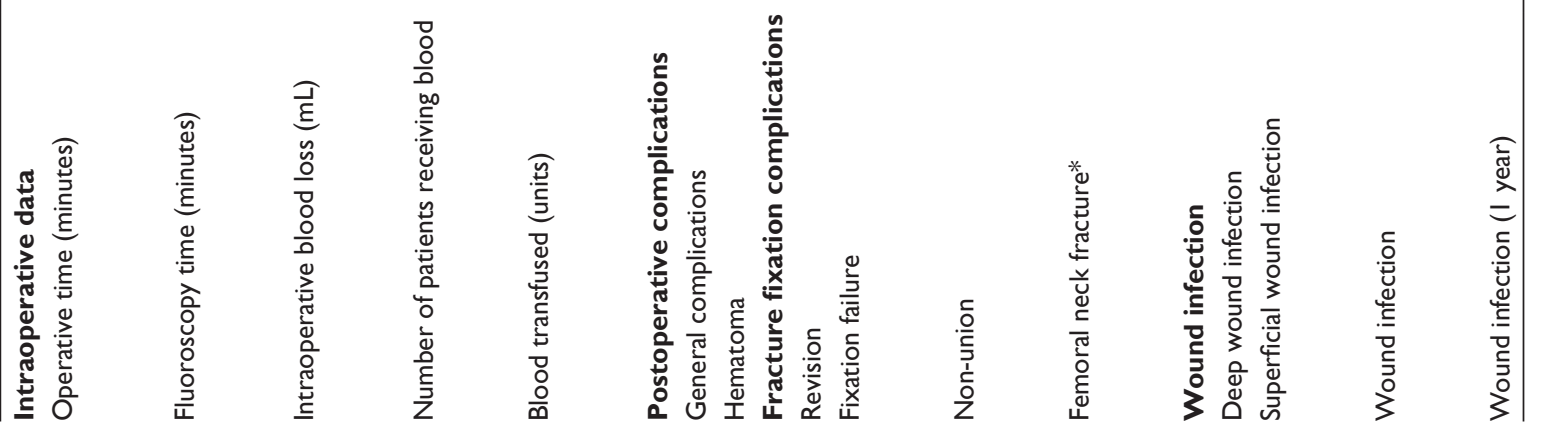




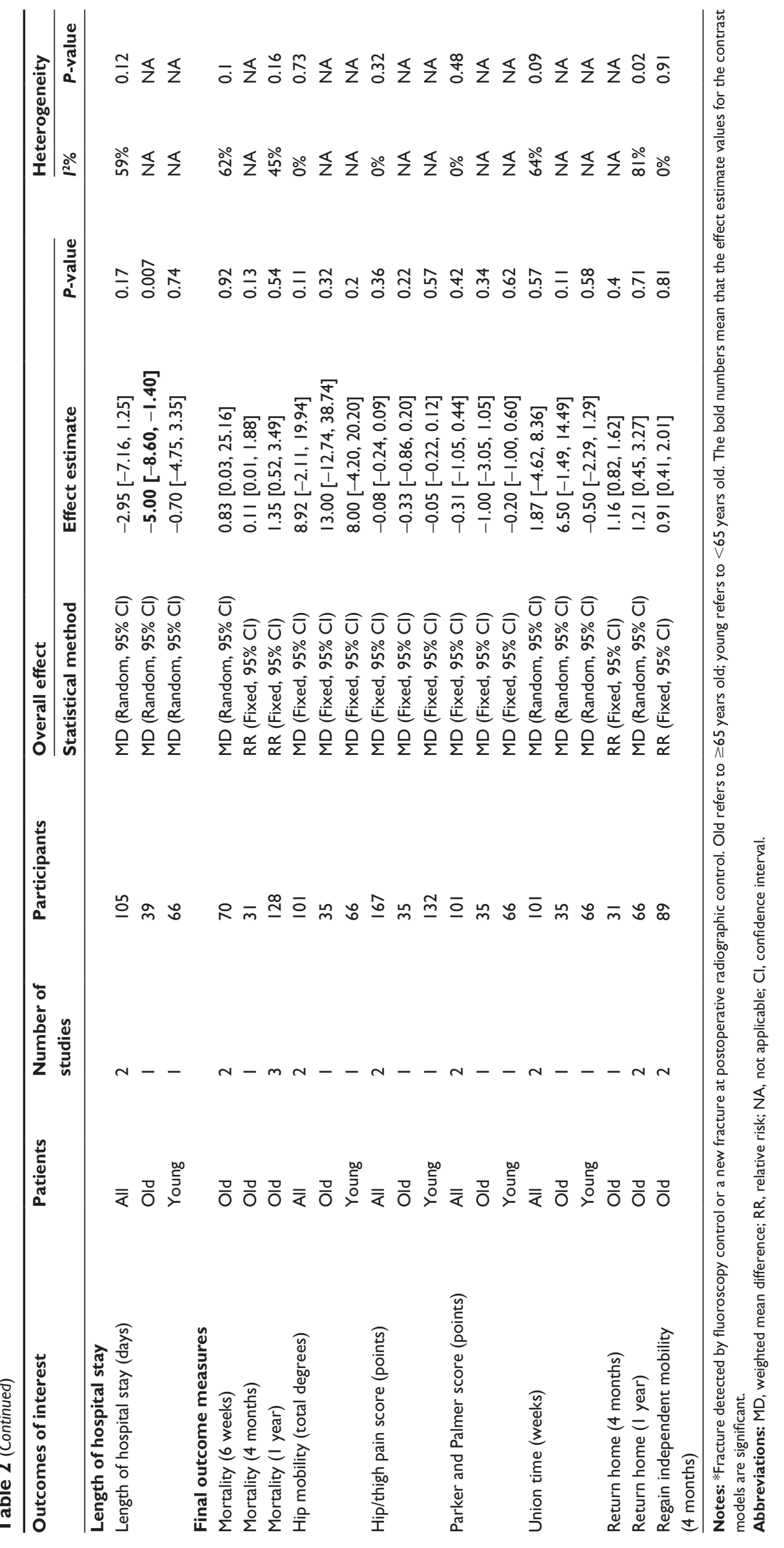




\begin{tabular}{|c|c|c|c|c|c|c|c|c|c|c|c|}
\hline \multirow{2}{*}{$\begin{array}{l}\text { Study or } \\
\text { subgroup } \\
\text { Rahme and Harris }{ }^{15}\end{array}$} & \multicolumn{2}{|c|}{ Intramedullary } & \multicolumn{2}{|c|}{$\begin{array}{l}\text { Extramedullary } \\
\text { Events Total }\end{array}$} & Weight & \multicolumn{2}{|l|}{$\begin{array}{l}\text { Risk ratio } \\
\mathrm{M}-\mathrm{H} \text {, fixed, } 95 \% \mathrm{Cl}\end{array}$} & \multicolumn{4}{|c|}{$\begin{array}{l}\text { Risk ratio } \\
\mathrm{M}-\mathrm{H} \text {, fixed, } 95 \% \mathrm{Cl}\end{array}$} \\
\hline & 0 & 29 & 8 & 29 & $57.9 \%$ & $0.06(0.00,0.97)$ & & & & & \\
\hline Sadowski et al ${ }^{12}$ & 2 & 18 & 6 & 17 & $42.1 \%$ & $0.31(0.07,1.35)$ & & & & & \\
\hline Total $(95 \% \mathrm{Cl})$ & & 47 & & 46 & $100 \%$ & $0.17(0.05,0.60)$ & & & & & \\
\hline \multirow[t]{2}{*}{ Total events } & 2 & & 14 & & & & & & & & \\
\hline & & & & & & & 0.005 & 0.1 & 1 & 10 & 200 \\
\hline \multicolumn{7}{|c|}{$\begin{array}{l}\text { Heterogeneity: } \chi^{2}=1.26, d f=1(P=0.26) ; I^{2}=21 \% \\
\text { Test for overall effect: } Z=2.74(P=0.006)\end{array}$} & & $\begin{array}{l}\text { vors } \\
\text { edullary }\end{array}$ & & $\begin{aligned} \text { Fa } \\
\text { extram }\end{aligned}$ & \\
\hline
\end{tabular}

Figure 3 The forest plot compares two RCTs that included 93 elderly patients for the revision rate.

Notes: The $95 \% \mathrm{Cl}$ of the pooled RR is $0.05-0.60$. It showed that there was also a lower risk of revision rate for elderly patients with intramedullary fixation.

Abbreviations: RCTs, randomized controlled trials; $\mathrm{M}-\mathrm{H}$, Mantzel-Haenzel method; $d f$, degrees of freedom; Cl, confidence interval; RR, relative risk.

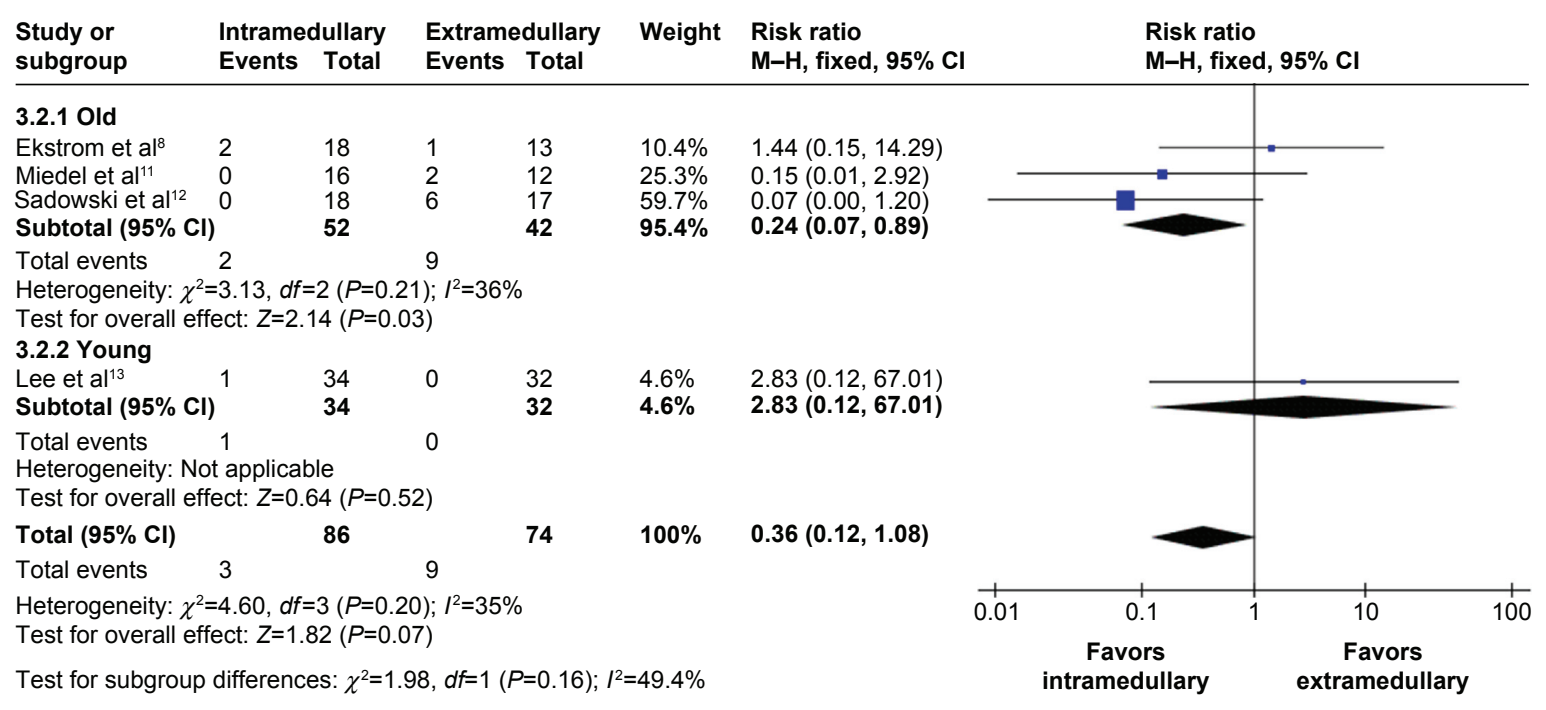

Figure 4 This forest plot shows the RR of fixation failure rate in four studies comparing intramedullary and extramedullary fixation, stratified by patient age. Notes: The diamonds indicate the $95 \% \mathrm{Cl}$ of the subgroup and the overall pooled estimate. It showed there was a significant lower risk of fixation failure rate for elderly patients with intramedullary fixation.

Abbreviations: $\mathrm{M}-\mathrm{H}$, Mantzel-Haenzel method; $d f$, degrees of freedom; $\mathrm{Cl}$, confidence interval; RR, relative risk.

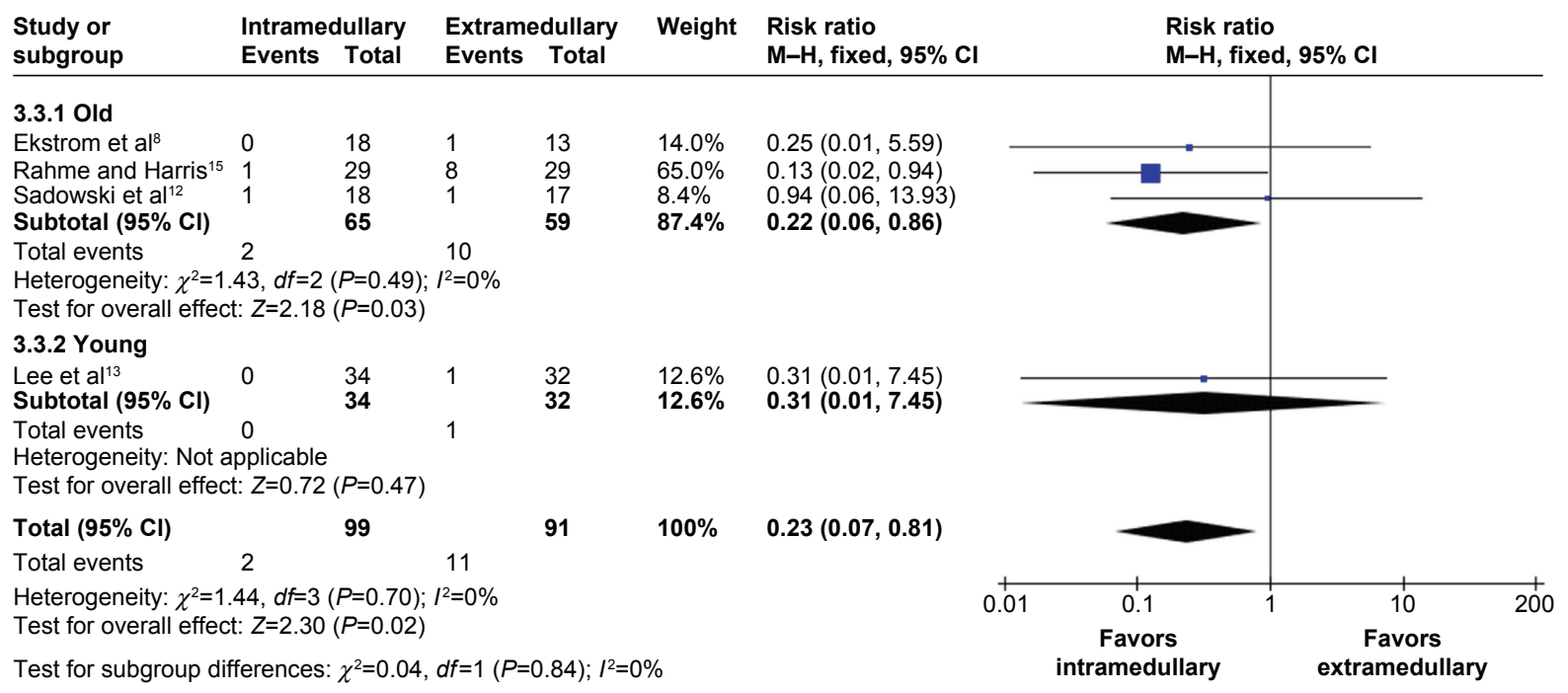

Figure $\mathbf{5}$ This forest plot shows the RR of non-union rate in four studies comparing intramedullary and extramedullary fixation, stratified by patient age.

Notes: The diamonds indicate the $95 \% \mathrm{Cl}$ of the subgroup and the overall pooled estimate. It showed there was a significantly lower risk of non-union rate for all patients with intramedullary fixation, especially in elderly patients.

Abbreviations: $\mathrm{M}-\mathrm{H}$, Mantzel-Haenzel method; $d f$, degrees of freedom; $\mathrm{Cl}$, confidence interval; RR, relative risk. 


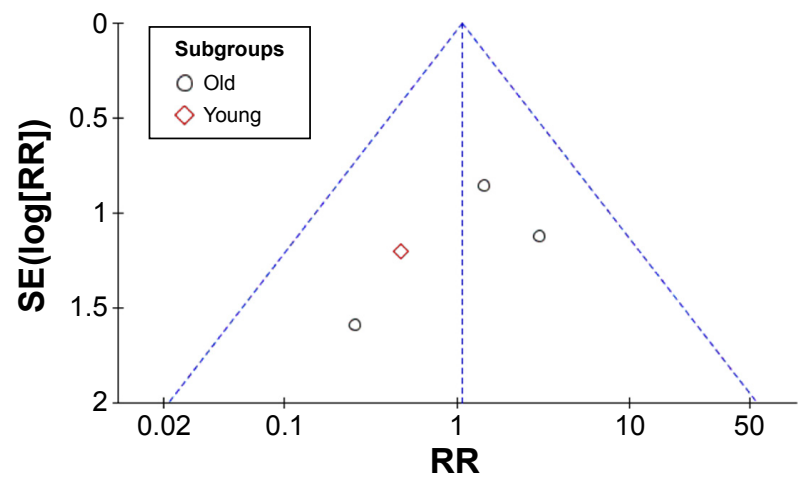

Figure 6 This funnel plot of four RCTs with 191 patients for wound infection shows no evidence of asymmetry, which suggests that there was no statistically significant publication bias. Data from Miedel et al, ${ }^{11}$ Sadowski et al, ${ }^{12}$ Lee et al, ${ }^{13}$ and Rahme and Harris. ${ }^{15}$

Abbreviations: RCTs, randomized controlled trials; RR, relative risk; SE, standard error.

non-union, $P=0.70, I^{2}=0 \%$; femoral neck fracture, $P=0.91$, $\left.I^{2}=0 \%\right)$.

No significant difference was observed between the intramedullary group and the extramedullary group for wound infection rate or length of hospital stay (days) when all of the patients were pooled into our meta-analysis. If significant heterogeneity was observed, the random-effects model was used because no significant clinical heterogeneity was observed between the studies.

There were no significant differences for final outcome measures in the extramedullary group: mortality (6 weeks, 4 months, 1 year); hip mobility (total degrees); hip/thigh pain score (points); Parker and Palmer score (points); union time (weeks); return home (4 months, 1 year); and regaining of independent mobility (4 months). If significant heterogeneity was observed, the random-effects model was used because no significant clinical heterogeneity was observed between the studies.

\section{Publication bias}

Funnel plots demonstrated no significant visual evidence of publication bias (Figure 6).

\section{Discussion}

This meta-analysis included six RCTs with small or medium sample sizes. The quality of the evidence in this review was moderate overall, but the small number of studies is a major threat to generalizability of our results, especially fixation implant differences.

Intramedullary devices provide more reliable fixation than extramedullary devices for subtrochanteric femur fractures. The revision rate was $83 \%$ lower for elderly patients in the intramedullary group compared with the extramedullary group. The fixation failure rate was $76 \%$ lower for elderly patients in the intramedullary group compared with the extramedullary group. The non-union rate was $77 \%$ lower for all patients, and it was $78 \%$ lower for elderly patients in the intramedullary group. However, there was no significant difference in femoral neck fracture rate. Therefore, a significant decrease occurred in the rate of fracture fixation complications for patients treated with intramedullary internal fixation, especially elderly patients. Therefore, we recommend that orthopedic physicians treat elderly subtrochanteric femur fractures using intramedullary internal fixation. The entire reason for this recommendation is the requirement of fewer reoperations. Reoperations are very expensive and are associated with high risk of morbidity and mortality.

No significant differences were found between the intramedullary group and the extramedullary group for intraoperative data, postoperative complications, wound infection rate, hospital stay days, and final outcome measures. Therefore, the reason why the final outcome measures do not change when the fracture fixation complication rates are so different is not known. Table 2 shows that intraoperative data (such as operative time, intraoperative blood loss and blood transfused units) were reduced compared with the extramedullary group, and fracture fixation complications were significantly reduced compared to the extramedullary group. Wound infections (such as deep wound infection and 1 -year wound infection rates) were reduced compared with the extramedullary group, and hospital stay days and final outcome measures (such as 6-week mortality, 4-month mortality, hip mobility degrees, hip/thigh pain scores, and return home rate) were better compared with the extramedullary group. The lack of differences in most categories is foreseeable. The patients ultimately reached the same recovery point in the final follow-up, but the course of patients who underwent revision surgeries, fixation failures, and non-unions was substantially more complicated and time-consuming and these factors likely resulted in interim decrements in outcome measures. The intramedullary group may have included longer fluoroscopy times, more patients who received blood, more superficial wound infection risks, more general complications (such as heart failure) and lower Parker and Palmer scores. These reasons may underlie the higher 1-year mortality rate and the regaining of independent mobility in 4 months. These differences were not significant, but the treatment of elderly subtrochanteric femur fractures using intramedullary internal fixation is recommended.

This meta-analysis included all studies of extensions into the subtrochanteric region. All studies included AO Foundation/Orthopaedic Trauma Association 31-A3 fractures because of the relatively rare occurrence of pure subtrochanteric femur fractures. ${ }^{2}$ This meta-analysis included 
the following limitations. First, the internal fixation implants in each trial were not perfectly identical, which may influence the outcomes of interest. Second, some parameters of interest demonstrated a large degree of heterogeneity. The heterogeneity of intraoperative data, wound infection, hospital stay days, and final outcome measures may be the result of bias from different surgical doctors in the trials. Lastly, this meta-analysis requires more patients than the few patients who were included, and larger level studies are required to show the superiority of intramedullary devices for subtrochanteric femur fractures between young high-energy trauma and elderly low-energy trauma.

In conclusion, the meta-analysis suggests that there was no significant difference in the intraoperative data, postoperative complications, wound infection, hospital stay days or final outcome measures between intramedullary and extramedullary internal fixation. However, a significant decrease occurred in the rate of fracture fixation complications for patients who were treated with intramedullary internal fixation, especially in elderly patients. Some differences were not significant, but the treatment of elderly subtrochanteric femur fractures using intramedullary internal fixation is recommended.

\section{Acknowledgments}

The authors gratefully acknowledge the support of the subjects who participated in the present study. The study was partly supported by the Natural Science Foundation of Shanghai, China (grant no 14ZR1432600).

\section{Disclosure}

The authors report no conflicts of interest in this work.

\section{References}

1. Tornetta P 3rd. Subtrochanteric femur fracture. J Orthop Trauma. 2002; 16(4):280-283.

2. Kuzyk PR, Bhandari M, McKee MD, Russell TA, Schemitsch EH. Intramedullary versus extramedullary fixation for subtrochanteric femur fractures. J Orthop Trauma. 2009;23(6):465-470.

Clinical Interventions in Aging

\section{Publish your work in this journal}

Clinical Interventions in Aging is an international, peer-reviewed journal focusing on evidence-based reports on the value or lack thereof of treatments intended to prevent or delay the onset of maladaptive correlates of aging in human beings. This journal is indexed on PubMed Central, MedLine,
3. Mahomed N, Harrington I, Kellam J, et al. Biomechanical analysis of the Gamma nail and sliding hip screw. Clin Orthop Relat Res. 1994;(304): 280-288.

4. Kraemer WJ, Hearn TC, Powell JN, Mahomed N. Fixation of segmental subtrochanteric fractures. A biomechanical study. Clin Orthop Relat Res. 1996;(332):71-79.

5. Roberts CS, Nawab A, Wang M, Voor MJ, Seligson D. Second generation intramedullary nailing of subtrochanteric femur fractures: A biomechanical study of fracture site motion. J Orthop Trauma. 2002;16(4): 231-238.

6. Kummer FJ, Olsson O, Pearlman CA, etal. Intramedullary versus extramedullary fixation of subtrochanteric fractures. A biomechanical study. Acta Orthop Scand. 1998;69(6):580-584.

7. Curtis MJ, Jinnah RH, Wilson V, Cunningham BW. Proximal femoral fractures: A biomechanical study to compare intramedullary and extramedullary fixation. Injury. 1994;25(2):99-104.

8. Ekstrom W, Karlsson-Thur C, Larsson S, Ragnarsson B, Alberts KA. Functional outcome in treatment of unstable trochanteric and subtrochanteric fractures with the proximal femoral nail and the Medoff sliding plate. J Orthop Trauma. 2007;21(1):18-25.

9. Matre K, Vinje T, Havelin LI, et al. TRIGEN INTERTAN intramedullary nail versus sliding hip screw: A prospective, randomized multicenter study on pain, function, and complications in 684 patients with an intertrochanteric or subtrochanteric fracture and one year of follow-up. J Bone Joint Surg Am. 2013;95(3):200-208.

10. Saarenpaa I, Heikkinen T, Jalovaara P. Treatment of subtrochanteric fractures. A comparison of the Gamma nail and the dynamic hip screw: Short-term outcome in 58 patients. Int Orthop. 2007;31(1):65-70.

11. Miedel R, Ponzer S, Tornkvist H, Soderqvist A, Tidermark J. The standard Gamma nail or the Medoff sliding plate for unstable trochanteric and subtrochanteric fractures. A randomised, controlled trial. J Bone Joint Surg Br. 2005;87(1):68-75.

12. Sadowski C, Lubbeke A, Saudan M, et al. Treatment of reverse oblique and transverse intertrochanteric fractures with use of an intramedullary nail or a 95 degrees screw-plate: A prospective, randomized study. J Bone Joint Surg Am. 2002;84-A(3):372-381.

13. Lee PC, Hsieh PH, Yu SW, et al. Biologic plating versus intramedullary nailing for comminuted subtrochanteric fractures in young adults: A prospective, randomized study of 66 cases. J Trauma. 2007;63(6): 1283-1291.

14. Goldhagen PR, O'Connor DR, Schwarze D, Schwartz E. A prospective comparative study of the compression hip screw and the gamma nail. J Orthop Trauma. 1994;8(5):367-372.

15. Rahme DM, Harris IA. Intramedullary nailing versus fixed angle blade plating for subtrochanteric femoral fractures: A prospective randomised controlled trial. J Orthop Surg (Hong Kong). 2007;15(3):278-281.
Dovepress

CAS, Scopus and the Elsevier Bibliographic databases. The manuscript management system is completely online and includes a very quick and fair peer-review system, which is all easy to use. Visit http://www.dovepress. com/testimonials.php to read real quotes from published authors. 